\title{
Diagnóstico, aproximación terapéutica y rehabilitación del alcohólico.
}

\author{
Ceccanti, M.; Balducci, G.; Attilia, M.L.; M. Romeo, M. \\ Centro di Riferimento Regionale delle Patologie e delle Problematiche Alcohol-Correlate Departamento de Medicina Clínica - Universidad “La Sapienza”, ROMA.
}

Resumen: El abuso de alcohol determina problemas y patología de gran importancia sanitaria y social. Se calculó que en los países occidentales, donde se vienen registrando sistemáticamente la incidencia y la prevalencia, el alcoholismo alcanza al $9 \%$ de la población con una edad superior a los catorce años. Alrededor del 20 al $40 \%$ de los pacientes tratados en hospitales tendrían problemas relacionados con el abuso de alcohol. La aproximación terapéutica a estos pacientes debe tener en cuenta los múltiples factores que determinan el alcoholismo (biológicos, psíquicos, sociales, etc.). Se deberá en primer lugar tratar el síndrome de abstinencia, caso de producirse. En esta primera fase es importante iniciar la acogida, que debe ser dirigida a establecer un contrato terapéutico duradero con el paciente. Debe hacerse un seguimiento riguroso de las variables bio-psico-sociales para valorar las distintas alternativas terapéuticas que van desde la intervención breve hasta actuaciones más especializadas como psicoterapia individual o grupal hasta las terapias farmacológicas anticraving. También se abordan las cuestiones referentes al seguimiento y a la prevención de las recaídas.

Palabras clave: alcoholismo, diagnóstico, tratamiento, rehabilitación, problemas relacionados.

\section{INTRODUCCIÓN.}

$\mathbf{E}$ I abuso de bebidas alcohólicas se considera unánimemente uno de los factores que, más frecuentemente, desencadenan graves problemas para la salud del hombre $(1,2)$. Efectivamente, el alcoholismo es un fenómeno que parece aumentar constantemente en la mayor parte de los países del mundo, si bien con un predominio y con unos costes sociales que varían ampliamente según el contexto en el que se manifieste.

Desde un punto de vista clínico, el término de "Patologías y Problemas Relacionados con el Alcohol" (PPAC) se refiere a todo el conjunto de fenómenos bio-psico-sociales causados por el abuso del alcohol que requieren un tratamiento específico de tipo "integrado" (médico, psicológico y de rehabilitación) (3).

Las manifestaciones de los PPAC pueden ser agudas o presentarse en desarrollos crónicos, según un espectro de variables que van desde sintomatologías leves a patologías muy graves, y por ello las respues-
Summary: The abuse of alcohol determines problems and pathologies of serious health and social significance. In the western countries, where there is a systematic registration of the incidence and prevalence of alcoholism, it is calculated that it affects $9 \%$ of the population over fourteen years of age. Around $20-40 \%$ of patients treated in hospital will be those with alcohol abuse-related problems. The therapeutic approach to these patients must take into account the multiple factors which determine alcoholism (biological, psychic, social, etc.). In the first place, there should be treatment of the withdrawal syndrome, when this occurs. The contact phase is very important, and must be aimed at establishing a durable therapeutic understanding with the patient. There must be a strict follow-up of the bio-psycho-social variables in order to evaluate the different therapeutic alternatives which range from the brief intervention to more specialised actions such as individual or group psychotherapy and include the anticraving pharmacological therapies. In addition, there should be an approach to such subjects as follow-up and prevention of relapses.

Key words: alcoholism, treatment interventions, psychotherapy, pharmacology, correlated problems, Italy.

tas a los tratamientos son igualmente muy variadas. Estas características obligan, por lo tanto, a los especialistas del sector a una gestión del paciente cuidadosamente programada, que ha de ser, necesariamente, "longitudinal."

Las tareas de los Servicios de Alcoholismo consisten, por consiguiente, en:

- La vigilancia epidemiológica del territorio, a través de un rastreo cuidadoso y una correcta y oportuna caracterización y definición del cuadro diagnóstico del paciente alcohólico;

- El establecimiento de pautas de educación de la salud del paciente y de sus familiares;

- Unas propuestas de intervenciones terapéuticas y de rehabilitación del alcoholismo.

\section{HÁBITOS DE BEBIDAY CUADRO DIAGNÓSTICO}

Una persona se considera Abstinente cuando en el transcurso de su vida nunca ha ingerido bebidas que 
contengan ninguna cantidad de alcohol. El resto deben ser considerados Bebedores (ocasionales, habituales, moderados, problemáticos o "de riesgo").

Es importante, sin embargo, subrayar que en la categoría de los que denominamos Bebedores existe una gran diversidad en lo que respecta al consumo de alcohol y hábitos de bebida. Por ello debe tenerse en cuenta que la Organización Mundial de la Salud (O.M.S.) aconseja no superar la dosis diaria de 40 g/día de alcohol en el hombre y de 25 g/día en la mujer (4). Estas dosis corresponden a las cantidades que se reflejan en la tabla que figura a continuación (Tab. 1).

\section{Tabla 1: Consumo alcohólico diario de bajo riesgo}

$\begin{array}{lrr} & \text { HOMBRE } & \text { MUJER } \\ \text { Vino }\left(10^{\circ} \text { vol\%) }\right. & 500 \mathrm{ml} & 300 \mathrm{ml} \\ \text { Cerveza }\left(4^{\circ} \text { vol\%) }\right. & 1200 \mathrm{ml} & 800 \mathrm{ml} \\ \text { Licores }\left(40^{\circ} \text { vol\%) }\right. & 135 \mathrm{ml} & 90 \mathrm{ml}\end{array}$

Fuente: O.M.S. (1993)

Tomando estos porcentajes como guía, podemos establecer una primera distinción, a grosso modo, entre:

- Bebedores moderados (los que beben por debajo de los niveles consignados);

- Bebedores "de riesgo" (los que beben por encima de esos niveles)

Aunque los problemas que se derivan de las diferencias (genético-ambientales) de los sistemas enzimáticos del metabolismo del alcohol etílico hacen extremadamente variable la susceptibilidad de un individuo a los efectos tóxicos del alcohol, hay un dato que parece ser irrefutable: el aumento del consumo de alcohol está fuertemente relacionado con el aumento de los riesgos para la salud $(1,2)$. Los resultados de dos extensos estudios americanos $(5,6)$ que han evaluado alrededor de 300.000 sujetos de sexo masculino han demostrado un aumento de la mortalidad en aquellos que consumían diariamente más de 2-3 unidades alcohólicas (U.A.) ${ }^{1}$. Otro estudio que se centraba en personas de sexo femenino (7) ha demostrado que las mujeres que beben diariamente cerca de 2,5 U.A. presentaban una mortalidad mucho más elevada respecto a las que bebían menos o no bebían en absoluto. Las diferencias de "tolerancia" al alcohol de las mujeres respecto a los hombres se deben, al parecer, y por lo menos en parte, al menor volumen de distribución corporal del etanol. También, en cierta medida, a la ausencia de la isoenzima gástrica del alcohol dehidrogenasa. Un estudio epidemiológico nacional sobre el estado de salud y los hábitos nutricionales de la población estadounidense (8) centrado en sujetos que referían excesos esporádicos superiores a las 4 U.A. y 8 U.A. en cada ocasión (en comparación con los abstemios) ha permitido concluir que los excesos ocasionales se asocian a un riesgo relativo de fallecimientos por accidente comprendido, respectivamente, entre el 1.9 y el $3.3 \%$. El "National Institute on Alcohol Abuse and Alcoholism" (NIAAA, USA) define el beber "moderadamente" como: "la cantidad media de consumo alcohólico diario que se asocia al menor riesgo de aparición de problemas relacionados con el alcohol" (9). Aunque ese criterio (que se aproxima de alguna manera a la definición de "bebedor de riesgo" de la O.M.S.) permita una estimación del riesgo para la salud de la población, no podemos minusvalorar el hecho de que los factores individuales, como la duración del consumo, pueden influir, y no poco, en los niveles de riesgo. Aunque existen evidencias sustanciales que subrayan que el beber moderadamente (cuando se compara con el no beber) puede asociarse a un cierto beneficio para la salud (por ejemplo, una reducción en los riesgos de mortalidad por enfermedades cardiovasculares), los niveles de consumo posibles son, sin embargo, notablemente bajos (no más de 2-6 U.A. por semana) (5). Un reciente estudio americano en sujetos de mediana edad y ancianos habría demostrado, en realidad, que los mayores beneficios, tanto en hombres como en mujeres, se obtenían con consumos de no más de una U.A. al día (10). De todos modos, debe precisarse que los beneficios generales sobre la mortalidad eran muy escasos y que, por otra parte, sólo podían considerarse completamente favorables en los sujetos más ancianos o en pacientes con cardiopatías (11). Por lo tanto, no existen, por el momento, evidencias definitivas a favor de la "prescripción" médica de alcohol, ni en términos "terapéuticos" (el anciano con cardiopatías) ni "preventivos".

Por lo que se refiere al cuadro diagnóstico, los criterios más utilizados internacionalmente son los que aparecen en el "Manuale Diagnostico e Statístico dei Disordini Mentali" (DSM) [Manual Diagnóstico y Estadístico de los Desórdenes Mentales], editado por la Asociación de Psiquiatría Americana (APA), del que actualmente existe una cuarta edición (APA; 1996) (12), que sitúa el alcoholismo entre las "Alteraciones

\footnotetext{
1 Unidad Alcohólica (U.A.). Cuando bebemos una bebida alcohólica de cualquier tipo (por ejemplo, vino, cerveza, licores, etc.) usando el recipiente que normalmente se utiliza para servirlas (por ejemplo, un vaso de $130 \mathrm{ml}$ para el vino, una jarra de $400 \mathrm{ml}$ para la cerveza o un vasito de $40 \mathrm{ml}$ para el aguardiente, etc.), ingerimos (aproximadamente) una cantidad de alcohol anhídrido equivalente (más o menos) a 12 g/etanol. Es posible evaluar, por lo tanto, más fácilmente el consumo de los alcoholes referidos considerando cada una de las cantidades expresadas más arriba equivalentes a 1 U.A., sumándolas después entre ellas y, multiplicándolas por 12, convertirlas en gramos de etanol.
} 
por uso de Sustancias", distinguiendo además dos modalidades diversas: Abuso y Dependencia.

\section{EPIDEMIOLOGÍA.}

\section{Investigaciones epidemiológicas sobre la pobla- ción general.}

Un estudio epidemiológico llevado a cabo en USA (1992) ha revelado que aproximadamente el 34\% de los adultos de la población es, en términos generales, completamente abstemio; el $44 \%$ bebe habitualmente y el $22 \%$ ha declarado haber bebido con anterioridad (13). Las estimaciones, siguiendo con datos procedentes de USA, reflejan un predominio de abuso y de dependencia del alcohol que varía, en las diversas casuísticas, del $7.4 \%$ al $9.7 \%(14,15)$ y un mayor porcentaje para los consumos que se refieren al transcurso de la vida completa que oscila entre el $13.7 \%$ y el $23.5 \%$ (15). Aunque el sexo masculino es el más directamente afectado por estos desórdenes y los consumos más elevados suelen a evaluarse en términos inversamente proporcionales a la edad, la renta y la escolaridad, ningún factor socio-demográfico parece ejercer un rol significativamente protector; todo el mundo está pues en condiciones de verse afectado por eventuales problemas relacionados con el alcohol.

\section{Investigaciones epidemiológicas en ámbitos clíni- cos.}

Los porcentajes de los casos de abuso y de dependencia del alcohol son generalmente mayores en el ámbito clínico. Esta afirmación parece confirmada por recientes observaciones desarrolladas en USA que demostrarían que aproximadamente el $20 \%$ de los pacientes de hospitales pueden ser adscritos a diagnósticos de alcoholismo (17). Otros autores (18) han observado un idéntico predominio del abuso y la dependencia del alcohol en las personas hospitalizadas, en torno al $20 \%$. En diversos estudios realizados en ambulatorios también se demuestra un predominio del alcoholismo comprendido entre el $16 \%$ y el $36 \%$ (19-21).

En nuestro país no existen datos que se refieran a la totalidad del territorio nacional. Sin embargo, nuestro grupo de investigación desarrolló en 1993, una investigación epidemiológica (Proyecto "TELEALCO", de Ceccanti y otros, 1993) sobre pacientes ingresados en Urgencias, en los dispensarios y en los departa- mentos de Medicina General de cinco grandes hospitales de la región del Lazio.

En la investigación se trabajó con 2740 pacientes (1721 M, 1019 F), de edades comprendidas entre los 14 y 90 años y un promedio de consumo de alcohol entre 0 y $768 \mathrm{~g} /$ día.

Todos los pacientes fueron sometidos a una cuidadosa anamnesis con el fin de evaluar las distintas modalidades de consumo de bebidas alcohólicas, y se les proporcionó un cuestionario de autoevaluación para establecer diagnósticos de abuso y de dependencia del alcohol (un test MAST, "Michigan Alcoholism Screening Test"). También se les pasaron los siguientes análisis de laboratorio habituales: MCV, GGT, AST, ALT.

Uno de los objetivos más importantes de la investigación era, entre otros, el de valorar el predominio del alcoholismo crónico en el número completo de pacientes ingresados por cualquier tipo de patología en los entes hospitalarios implicados en la iniciativa. Los resultados de este estudio demostraron que entre los que referían elevados consumos de riesgo por PPAC no existían diferencias importantes derivadas de la escolarización y los oficios: estos sujetos representaban el $27.5 \%$ de la población estudiada. En cambio, según los resultados del MAST, el 22\% obtenía una puntuación que se podría asociar a condiciones de abuso y/o dependencia del alcohol. En la Tabla $\mathrm{n}^{\circ} 2$ se recogen los motivos que habían declarado al ingresar los pacientes estudiados, en los que no puede observarse una asociación sistemática entre alcoholismo y patología orgánica.

\section{Tabla 2: DIAGNÓSTICOS DE ENTRADA}

$\begin{array}{lrr}\text { Patología } & \begin{array}{r}\text { Total } \\ (\%)\end{array} & \begin{array}{r}\text { Alcohólicos } \\ (\%)^{2}\end{array} \\ \text { Cardiovasculares } & 41,2 & 20,3 \\ \text { Respiratorias } & 15,3 & 26,2 \\ \text { Gastrointestinales } & 14,2 & 12,5 \\ \text { Neurológicas y psiquiátricas } & 17,8 & 12,2 \\ \text { Hepáticas } & 11,3 & 22,6 \\ \text { Dismetabólicas } & 7,3 & 5,0 \\ \text { Osteoarticulares } & 6,6 & 22,2 \\ \text { Neoplásicas } & 6,6 & 11,1 \\ \text { Hemopoyéticas } & 2,1 & 16,6 \\ \text { Hiperpiréticas } & 4,7 & 15,4 \\ \text { Otras } & 7,3 & 10,0 \\ \text { Alcoholismo } & 5,5 & \end{array}$

Alcoholismo

5,5

Fuente: Proyecto TELEALCO (Ceccanti et al., 1993).

2 Porcentaje de sujetos con consumo de alcohol de riesgo en relación con la patología considerada en cada caso. 
PROBLEMAS DE DIAGNÓSTICOY DE RASTREO.

En muchos países, industrializados o no, a pesar de la alta incidencia de las PPAC, en un elevado porcentaje de casos no existe un diagnóstico de alcoholismo.

En un estudio llevado a cabo en los USA, los médicos sólo establecieron correctamente un diagnóstico de alcoholismo en menos de la mitad de los pacientes (17) y en otro estudio, sólo se recomendó seguir un tratamiento al $24 \%$ de los pacientes alcohólicos (18).

En Italia, aunque se estima que el diagnóstico de alcoholismo en presencia de patologías médicas concomitantes (las llamadas patologías relacionadas con el alcohol) es más frecuente, no siempre parece que este axioma se satisfaga. En el ámbito del proyecto "TELEALCO", sólo un 5,5\% de los casos fue diagnosticado expresamente como alcoholismo crónico por el personal sanitario implicado en el estudio, frente a un predominio de los bebedores "de riesgo" del 27,5\%, y un índice de respuesta positiva al MAST del 22\%.

En el caso de las mujeres, los especialistas del sector se enfrentan a otro serio problema, en este caso porque las posibilidades de caracterización individualizada del diagnóstico parecen ser, definitivamente, más difíciles que en los hombres.

En la base de esta escasa capacidad de caracterización, que afecta a amplios estratos de especialistas socio-sanitarios, se encuentran numerosos factores, si bien los más importantes pueden relacionarse con la falta de adecuación de la formación universitaria y profesional (23), el escepticismo de muchos acerca de la eficacia de los programas terapéuticos disponibles (17) y la idea de que el alcoholismo no pertenece al campo de acción de la medicina clínica (24). Y, sin embargo, en los comportamientos alcohólicos "de riesgo" la detección precoz constituye un arma irrenunciable para la prevención de las secuelas a largo plazo. Todo esto hace que la fase de detección y encuadre diagnóstico sea realmente "crítica" en el ámbito del conjunto de las relaciones entre médico y paciente.

\section{LAS PRIMERAS MEDIDAS TERAPÉUTICAS EN EL TRATAMIENTO DEL ALCOHOLISMO.}

Después de la identificación y del encuadre diagnóstico el primer objetivo de los especialistas del sector es el de "enganchar" al paciente con el fin de que desarrolle un cambio en su estilo de vida personal, o sea, en sus comportamientos habituales. Algunas veces, sobre todo en los casos de simple abuso de bebidas alcohólicas, puede ser suficiente que el personal sanitario le proporcione información correcta acerca de los riesgos que conlleva el beber en los llamados casos "de riesgo", acompañado de breves sesiones de consejos para la motivación (por ejemplo, una 'intervención breve' de 3 a 5 sesiones en total). En otros casos puede llegar a ser necesario recurrir a la hospitalización y a la aplicación de un proceso terapéutico y de rehabilitación más complejo, de tipo integrado, como ocurre en los casos graves de dependencia del alcohol. En definitiva, un atento seguimiento del paciente alcohólico puede favorecer, en un continuo "feedback" entre paciente y especialista, la adecuación constante de la intervención terapéutica con una atenta monitorización de la respuesta del alcohólico al tratamiento.

\section{RESPUESTA Y VALORACIÓN DE LA “RAPIDEZ DEL CAMBIO".}

Cuando se ha llegado al reconocimiento de un problema de alcoholismo en un paciente, es verdaderamente decisivo que se pase a una segunda fase del diagnóstico, que es importantísima, y que es la que se ocupa de la valoración del nivel de percepción que existe en el sujeto sobre la existencia de un verdadero problema de alcoholismo. De hecho, muchos pacientes no parecen llegar a considerar correctamente las relaciones de causa-efecto existente entre el abuso del alcohol y los problemas que sufren y que están relacionados con ello. Muchas veces esta negación tiene su origen en el deseo de esconder el sentimiento de vergüenza que está experimentando, junto a sensaciones de descenso en la autoestima y desconfianza su propia capacidad para poder llevar a cabo el cambio. Los especialistas deben por ello mostrarse sensibles hacia los sentimientos del paciente, tratando, al mismo tiempo, de convencer al alcohólico, evitando juzgarlo, y de concienciarle de que reconocer la existencia de un problema con el alcohol es especialmente importante para el éxito terapéutico.

En una primera fase de conversaciones se pueden utilizar las provechosas informaciones anamnésicas que ha proporcionado el propio paciente, los datos del reconocimiento médico objetivo y los análisis llevados a cabo en los laboratorios, para intentar aclararle el papel que ha jugado en todo ello el alcohol, enfatizando las ventajas que podrían derivarse en el caso de que decidiese dejar la bebida.

En el modelo de las "Seis fases del cambio" de Prochaska y de DiClemente (25) se subraya que la valoración de las fases del cambio de los comportamientos de dependencia es un buen índice para predecir el desarrollo del proceso terapéutico y del éxito del tratamiento. Por otra parte, el conocimiento de la "fase de cambio" en la que se encuentra el paciente puede permitir una mejor intervención del especialis- 
ta, que puede recurrir a las medidas específicas diseñadas ex profeso para cada una de las etapas $(25,26)$.

Por ejemplo, independientemente de la habilidad del especialista, algunos pacientes niegan categóricamente que el alcohol sea la causa de sus problemas. Se trata de los llamados pacientes "Precontemplativos". La única intervención que es posible llevar a cabo en estos casos de individuos tan reacios a la colaboración es intentar, por lo menos, "engancharles", aunque sea con medidas de carácter eminentemente médico (porque muchos de ellos tienen evidentes patologías relacionadas con el alcohol), para intentar, con el tiempo, en los controles ambulatorios sucesivos, conseguir la superación de la negación (toma de conciencia por parte del sujeto), trasladando al alcohólico a una segunda fase de cambio que se denomina "fase de contemplación" (reconocimiento de la existencia de un problema de dependencia del alcohol). Algunas veces es el mismo paciente el que manifiesta abiertamente un deseo de transformar su propio estado de dependencia ("fase de determinación"), una fase que puede considerarse muy próxima a otra en la que el paciente inicia verdaderamente un cambio en sus comportamientos ("fase de acción"). Seguidamente, el paso inmediatamente posterior en la evolución del sujeto es el que lo conduce a una fase específica, llamada de "mantenimiento" que es típica de aquellos pacientes que han dejado de beber pero que necesitan, de todos modos, someterse a unos controles periódicos para evaluar su situación médica, psicológica y de relación con los demás, con el fin de prevenir en la mayor medida posible (o evitar) eventuales "fases de recaída", así como para acordar conjuntamente, cuando sea preciso, intervenciones ocasionales de mantenimiento como pueden ser la asistencia a un grupo de auto-ayuda, el consejo motivacional, etc.

\section{“BRIEF INTERVENTION” EN LOS BEBEDORES “DE RIESGO".}

La eficacia terapéutica del asesoramiento conocido como 'intervenciones breves' en los bebedores "de riesgo" que no son dependientes del alcohol es clarísima. Este dato está perfectamente documentado en el ámbito internacional $(27,28)$.

La técnica que debe aplicarse en tales casos no parece ser excesivamente compleja, previendo, sustancialmente, que en el transcurso de las conversaciones entre médico y paciente el primero sea capaz de mostrar y hacer reconocer al segundo los estrechos vínculos que existen entre su "sentirse mal" y el abuso del alcohol, suministrando al mismo tiempo datos útiles sobre las dosis "de riesgo" e invitando al paciente a controlar mejor el uso de la sustancia.

\section{APROXIMACIONES TERAPÉUTICAS EN LOS PACIENTES CON DEPENDENCIA DEL ALCOHOL.}

En estos pacientes se requiere ciertamente un esfuerzo mayor por parte de los especialistas, de tipo multidisciplinar. Aunque el principal objetivo de la terapia en este caso sea ayudar al paciente a conseguir la sobriedad, la abstinencia del alcohol no siempre es completa y duradera. Los alcohólicos que son crónicos desde hace mucho tiempo están expuestos, en la mayor parte de los casos, a caídas o recaídas (lapse/relapse), y precisamente por ello los índices de éxito terapéutico que actualmente se consideran en el campo de la investigación clínica son los que se refieren a:

- la duración completa de la abstinencia (CAD) en relación con el número total de días de terapia (CTD):

- conseguir la sobriedad o, por lo menos, una reducción significativa del consumo;

- la mejora progresiva de las condiciones psicofísicas y el buen comportamiento social del individuo.

Algunos tratamientos pueden ser muy prolongados en el tiempo (a veces requieren años), sobre todo en lo que respecta a los controles que se llevan a cabo en los ambulatorios, que es necesario incrementar progresivamente cuando aparecen síntomas "guía", principalmente en forma de aumento del número de recaídas o por el agravamiento de una PPAC previa, etc. En algunos casos resulta posible conseguir la desaparición total del problema, incluso sin necesidad de ninguna intervención terapéutica, aunque esto, ciertamente, sólo suele darse en sujetos con una dependencia muy leve del alcohol. En algunos pacientes podría contemplarse la posibilidad de la vía de bebida moderada. Sin embargo, existe un dato incuestionable: en los sujetos con un problema grave de dependencia del alcohol, aquellos que sólo limitan el consumo, sin abandonarlo completamente, tienen un éxito terapéutico peor que los que se abstienen (29).

Una vez que el paciente alcohólico manifiesta el deseo de empezar el tratamiento y dejar de beber se programa una intervención articulada que contemple toda la problemática que la gestión de dicho proceso comporta. Lo más importante es que una de las primeras medidas que el especialista debe adoptar es la de decidir si se debe tratar farmacológicamente o no los posibles síntomas del Síndrome de Abstinencia del Alcohol (SAA), siempre al acecho. Deben también ponerse las bases, siempre en una primera fase, para preparar cuanto antes, si es posible, un tratamiento psico-social de apoyo, o, en el caso de que sea necesario, la aplicación de un tratamiento farmacológico de prevención de recaídas (tratamiento anti-craving). 
La importancia de esta fase inicial del tratamiento, precisamente por su contenido preventivo, puede intuirse a la luz de la morbilidad y la mortalidad relacionadas con el alcohol (30).

\section{GESTIÓN DEL SAA.}

La mayoría de los pacientes con síntomas leves de abstinencia (por ejemplo, temblor de manos por las mañanas) no requieren un tratamiento farmacológico específico. En muchos de estos casos, o incluso en aquellos más moderados, el seguimiento puede llevarse a cabo tranquilamente a nivel de ambulatorio (31). En general, la gravedad de los síndromes de abstinencia, su desarrollo y su respuesta al tratamiento pueden ser monitorizados adecuadamente recurriendo a escalas o baremos sencillos, como el "Clinical Institute Withdrawal Assessmet for Alcohol-revised" (CIWA-Ar) (32), que evalúa el desarrollo de los diez síntomas más frecuentes asociados al SAA. La importancia de estos índices radica sobre todo en su valor para orientar correctamente sobre la necesidad o no de proporcionar tratamientos farmacológicos, como puede verse en la Tabla 3.

El objetivo principal del tratamiento farmacológico del SAA es, obviamente, aliviar en la mayor medida posible la intensidad de los síntomas de abstinencia, intentando prevenir, al mismo tiempo, la aparición de manifestaciones clínicas especialmente críticas como las convulsiones, los desórdenes cerebrales derivados de la falta de nutrición (síndrome de Wernicke, síndrome de Korsakoff) o el Delirium tremens, uno de los síntomas más graves del SAA, por su relación con la mortalidad. La terapia debe conseguir, también, aumentar la conformidad del paciente en relación con el tratamiento, contribuyendo de ese modo al mantenimiento de la sobriedad. Un último objetivo, pero no por ello menos importante, que concierne al tratamiento farmacológico, es el de conseguir éxitos a largo plazo, aspecto éste que no suele recibir mucha atención por parte de los especialistas del sector.

Aunque los fármacos que se recomiendan para la gestión terapéutica del SAA van cambiando con el tiempo, las benzodiacepinas (BDZ) siguen siendo habitualmente las más habituales en muchos tratamientos, sobre todo por su eficacia, el bajo coste y su alto índice de seguridad (Tabla 5) (1,33). En una reciente publicación en la que se recogen los datos relativos a un meta análisis basado en los resultados obtenidos a partir de diferentes ensayos clínicos, se demuestra claramente que las BDZ reducen significativamente la incidencia de las convulsiones asociadas al SAA y la evolución del Delirium tremens (33). El Clordiazepóxido, el Diazepam, el Lorazepam y el Oxazepam son los agentes farmacológicos más usados en los centros para el tratamiento del alcoholismo de los USA (34). Las BDZ de vida media breve son, al parecer, más efectivas en pacientes con insuficiencia hepática grave. Todas las modalidades de posología (uso de dosis fijas, dosis iniciales de ataque o dosis ajustadas a la intensidad de la sintomatología) se han demostrado igualmente válidas para el control de los síntomas de abstinencia del alcohol (35).

Entre los fármacos más habituales últimamente destacamos (véase la Tabla 4) los beta-bloqueantes, la clonidina y la carbamazepina. De cualquier manera, la

\section{Tabla 3: Puntuación CIWA-Ar y Tratamiento farmacológico del SAA.}

Puntuación

$<10$ puntos

$10-20$ puntos

$>20$ puntos
Decisiones a adoptar

El paciente no necesita tratamiento.

El paciente debe ser observado durante un tiempo, repitiéndose eventualmente la valoración de los baremos.

El paciente debe someterse inmediatamente a la terapia farmacológica anti abstinencia.

Tabla 4: Fármacos anti-abstinencia utilizados frecuentemente en el tratamiento del SAA.

Benzodiazepinas

Beta bloqueantes

Alfa agonistas

Antiepilépticos
Reducen la gravedad de la abstinencia, estabilizan los signos vitales, previenen las convulsiones y el delirium tremens.

Mejoran los signos vitales, reducen el craving.

Reducen los síntomas de abstinencia.

Reducen la gravedad de la abstinencia, previenen las convulsiones.
Clordiazepoxido, Diazepam, Oxazepam, etc.

Atenolol, Propanolol

Clonidina

Carbamazepina 
capacidad que las BDZ tienen para prevenir las convulsiones durante el SAA determina que sigan siendo las preferidas en relación con otras posibilidades farmacológicas, sobre todo porque, además, los otros fármacos mencionados provocan más efectos secundarios, muchas veces de importancia.

Recientemente se ha incorporado al vademecum farmacéutico, en Italia, el ácido gamma-hidroxibutírico (GHB), un derivado del GABA, que desde los primeros estudios clínicos "piloto" ha demostrado poseer un gran interés científico en lo que respecta al tratamiento del SAA.

En un grupo de pacientes alcohólicos escogidos aleatoriamente entre los ingresados en nuestro Hospital de día son síntomas de SAA que necesitaban tratamiento farmacológico (con puntuación CIWA-Ar >20 puntos), se evaluó "a ciegas" la eficacia terapéutica del GHB respecto a una BDZ (Diazepam) (36). Los sujetos escogidos para la prueba fueron tratados durante un período de diez días completos, evaluándose la eficacia terapéutica del tratamiento mediante la ejecución de T0-T5, T10 y T18 del CIWA-Ar (para la valoración de la gravedad de los síntomas físicos de la abstinencia) y tests psico-métricos STAI-Y1 (para la valoración de los estados de ansiedad) y SDS de Zung (para la valoración de los estados de depresión). Los resultados de nuestro estudio figuran en la Tabla 5 y la Figura 1.
Los datos resultantes, obtenidos a partir de una muestra representativa de alcohólicos, parecen demostrar claramente que en el tratamiento farmacológico del SAA la eficacia del GHB es mayor que la de las BDZ. A esto hay que añadir, además, una ausencia completa de efectos colaterales, sin que tampoco hayan aparecido síntomas de dependencia del fármaco ni de fenómenos de efecto rebote asociables a la interrupción de la ingesta (36). Las dosis de GHB utilizadas son las aconsejadas $(50 \mathrm{mg} /$ por $\mathrm{kg} /$ día en tres tomas suministradas por boca) tres veces diarias durante un período de diez días. Entre las ventajas del GHB debe señalarse la mayor rapidez de actuación en una fase del tratamiento especialmente importante, que es la de los 3-4 primeros días del proceso terapéutico.

\section{PREVENCIÓN DE LAS RECAÍDAS Y MANTENI- MIENTO DE LA SOBRIEDAD.}

\section{Las terapias psicosociales de apoyo.}

Recientemente se han publicado los datos de un proyecto multicéntrico en el que han participado numerosos centros estadounidenses interesados en

Tabla 5: Ensayo clínico sobre la eficacia terapéutica del GHB vs. BDZ en alcohólicos con SAA.

\begin{tabular}{lcclllll} 
Grupo & T1 & T2 & \multicolumn{1}{c}{ T3 } & \multicolumn{1}{c}{ T4 } & \multicolumn{1}{c}{ T5 } & T10 & T18 \\
& $14,86 \pm$ & $9,71 \pm$ & $6,92 \pm$ & $6,50 \pm$ & $5,09 \pm$ & $3,62 \pm$ & $3,00 \pm$ \\
Gr. A & 6,8 & 6,8 & 4,68 & 7,04 & 3,59 & 2,50 & 2,73 \\
& 14,00 & $9,50 \pm$ & $5,13 \pm$ & $4,57 \pm$ & $5,00 \pm$ & $4,26 \pm$ & $3,17 \pm$ \\
Gr. B & $\pm 3,14$ & 4,31 & 4,14 & 2,90 & 3,05 & 4,15 & 3,21 \\
Gr. C & $5,45 \pm$ & $3,68 \pm$ & $3,71 \pm$ & $3,47 \pm$ & $2,78 \pm$ & $1,83 \pm$ & $2,94 \pm$ \\
& 3,10 & 2,79 & 2,59 & 2,29 & 2,51 & 1,47 & 2,77
\end{tabular}

Puntuación CIWA-Ar ( $m \pm$ SD). Gr. A (BDZ), Gr. B (GHB); Gr. C (sin tratamiento).

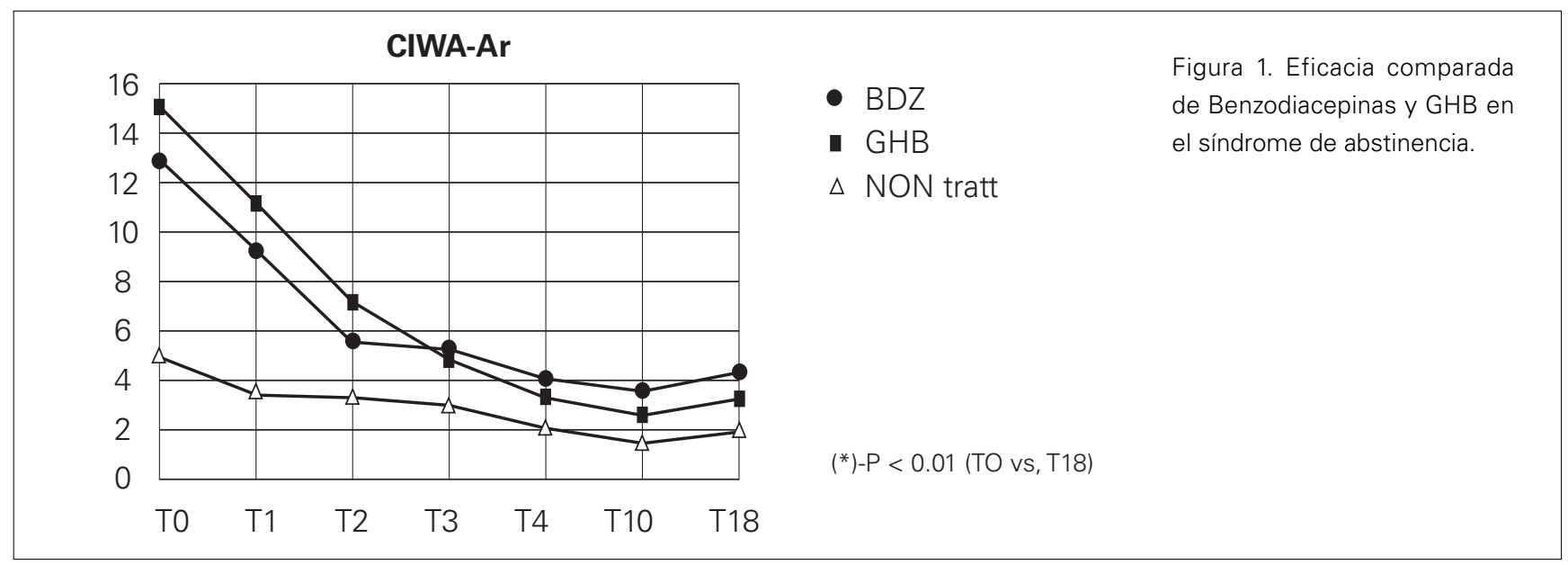


el tratamiento de las PPAC, cuyo objetivo era valorar la eficacia terapéutica de los diversos programas alcohólicos de apoyo que se ofrecen a los usuarios en los Servicios de Alcoholismo: los grupos de autoayuda, las técnicas cognitivas y de comportamiento (Auto-eficacia, terapia de técnicas de afrontamiento, etcétera) y el Counseling para la motivación

El estudio (Proyecto MATCH, Matching Alcoholism Treatment to Client Heterogenity) se ha llevado a cabo sobre un conjunto de 774 pacientes hospitalizados y 952 pacientes tratados en ambulatorios, que se repartieron aleatoriamente en tres grupos diferentes y se sometieron al tratamiento (37). Los indicadores de éxito terapéutico que se utilizaron para la evaluación de los datos se establecieron en base a dos niveles diferentes: en el nivel I, indicadores como el CAD y los consumos alcohólicos en cada ocasión; en el nivel II el tiempo de la primera caída (slip, primer vaso) y el tiempo de recaída (con un mínimo de tres días sucesivos de consumos "de riesgo").

En general el estudio ha demostrado que existen grandes y significativas diferencias en el éxito terapéutico anual entre los que habían recibido un tratamiento psico-social de mantenimiento, sin que puedan apreciarse diferencias significativas entre los diversos sistemas evaluados. Los pacientes hospitalizados presentaban un incremento, en lo que se refiere al CAD que pasó de un 20\% (en el mes anterior al tratamiento) a un $90 \%$ (en el mes siguiente al inicio del tratamiento). Después de doce meses el 35\% de los pacientes "hospitalizados" seguía manteniendo la sobriedad, el 25\% había estado expuesto a "caídas" y el resto, un 40\%, a "recaídas". En los pacientes "ambulatorios" el porcentaje del CAD se obtuvo en una media del $80 \%$ en el transcurso de los doce meses de seguimiento, con un $19 \%$ de individuos que se habían mantenido sobrios, un 35\% que había "caído" y un 46\% que había "recaído".

Independientemente de las cifras, los datos de este interesante estudio parecen poner de relieve la importancia del rol que otros factores parecen ejercer sobre el buen éxito terapéutico del tratamiento, factores que están representados sobre todo por la gravedad de los síntomas psiquiátricos que suelen asociarse (morbilidad psiquiátrica) al estado de motivación y la fase de cambio en la que se encuentre el paciente, al sexo (femenino) y a la gravedad de la sociopatía.

La gestión moderadamente más satisfactoria de los pacientes bajo tratamiento parece estar relacionada con la calidad de las posibilidades que se ofrecen, con una buena difusión sobre el territorio de los Centros implicados y con la disponibilidas de acceso, factores que aumentan en conjunto la compliance del paciente.

\section{PREVENCIÓN DE LAS RECAÍDAS.}

\section{Los fármacos anti-craving.}

Actualmente sólo existen dos fármacos aprobados por el FDA en los USA para el tratamiento terapéutico farmacológico anti-craving en la prevención de las recaídas del paciente alcohólico: el Disulfiram (un inhibidor de la conversión del acetaldehído en acetato) y la Naltrexona (un antagonista de los opiáceos que se toma por vía oral).

El craving puede ser definido como el "deseo del efecto conocido de una sustancia psicoactiva. Este deseo puede llegar a ser irresistible y puede aumentar en presencia de estímulos internos y externos (sucesos encadenados), y con la percepción de la disponibilidad de la sustancia. Se caracteriza por un comportamiento dirigido a la obtención de la sustancia $y$, en el ser humano, por pensamientos que se concentran en la misma" (Reunión de la UNDCP, Viena, 1992).

Actualmente existen también otros fármacos dirigidos a esta finalidad específica, aunque actualmente se encuentran en fase de experimentación. Entre ellos, uno de los más ampliamente estudiados en Europa es el Acamprosato (Homotaurinato de Calcio). Otros grupos de investigación han comenzado numerosos ensayos clínicos en "doble ciego" con fármacos del grupo de los inhibidores del recaptación de la serotonina, los antagonistas de la serotonina, los agentes GABEérgicos y Dopaminérgicos. En el estado actual de los conocimientos se subraya el hecho de que ninguno de los tratamientos habituales ha resultado ser completamente eficaz para la prevención de las recaídas, aunque la mayor parte de los ensayos clínicos reportan una eficacia entre el $20-35 \%$ en la persistencia de la sobriedad y de cerca del $50 \%$ en la garantía de una reducción significativa de los consumos de alcohol durante períodos de 12 a 14 meses de seguimiento en la fase inmediatamente posterior a la abstinencia $(38,39)$. La importancia de la "reducción del daño" en ese largo período hace que el argumento del control del craving sea muy interesante, y por lo tanto se puede predecir que la investigación farmacéutica sobre nuevas fórmulas farmacológicas que sean cada vez más eficaces continúen, como continúan paralelamente las investigaciones clínicas centradas en los métodos más idóneos para el control de las recaídas.

\section{BIBLIOGRAFÍA:}

(1) Secretary of Health and Human Services. Ninth special report to the U.S. Congress on Alcohol and health. 
Washington, D.C.: Government Printing Office, 1997. (NIH publication no. 97-4017).

(2) Lieber CS. Medical disorders of alcoholism. N Engl J Med 1995; 333: 1058-65

(3) Ceccanti M. et al.: Atti della I Consensus Conference sull'alcol. Scientific Press (Firenze); 1995.

(4) Saunders JB, Aasland OG, Babor TF, de la Fuente JR, Grant M. Development of the Alcohol Use Disorders Identification Test (AUDIT): WHO Collaborative Project on Early Detection of Persons with Harmful Alcohol Consumption - II. Addiction 1993; 88: 791-804.

(5) Camargo CA Jr, Hennekens CH, Gaziano JM, Glynn RJ, Manson JE, Stampfer MJ. Prospective study of moderate alcohol consumption and mortality in US male physician. Arch Intern Med 1997; 157: 79-85.

(6) Boffetta P., Gardinkel L. Alcohol drinking and mortality among men enrolled in an American Cancer Society prospective study. Epidemiology 1990; 1: 342-8.

(7) Fuchs CS, Stampfer MJ, Colditz GA, et al. Alcohol consumption and mortality among women. N Engl J Med 1995; 332: 1245-50.

(8) S. Anda RF, Williamson DF, Remington PL. Alcohol and fatal injuries among US adults: findings from the NHANES I Epidemiologie Follow-up Study. JAMA 1988; 260 : 2529-32.

(9) National Institute on Alcohol Abuse and Alcoholism. The physicians' guide to helping patients with alcohol problems. Washington, D.C.: Government Printing Office, 1995 (NIH publication no. 95-3769).

(10) Thun MJ, Peto R, Lopez AD, et al. Alcohol consumption and mortality among middle-aged and elderly U.S. adults. N Engl J Med 1997; 337: 1705-14.

(11) Porter JD. Hazards and benefits of alcohol. N Engl J Med 1997; 337: 1763-4.

(12) Diagnostic and statistical manual of mental disorders, 4" ed: DSM IV. Washington, D.C.: American Psychiatric Association, 1994.

(13) Dawson DA, Grant BF, Chou SP, Pickering RP. Subgroup variation in U.S. drinking patterns: results of the 1992 National Longitudinal Alcohol Epidemiologie Study. J Subst Abuse 1995; 7: 331-44.

(14) Grant BF.: prevalence and correlates of drug use and DSM-IV drug dependence in the United States: results of the National Longitudinal Alcohol Epidemiologie Survey. J Subst Abuse 1996; 8: 195-210.

(15) Kessler RC, Mc Gonagle KA, Zhao S, et al. Lifetime and 12 month prevalence of DSM - III - R psychiatric disorders in the United States: results from the National Comorbidity Survey. Arch Gen Psychiatry 1994; 51: 819.

(16) Helzer JE, Pryzbech TR. The co-occurrence of alcoholism with other psychiatric disorders in the general population and its impact on treatment J Stud Alcohol 1988; 49: 219-24.

(17) Moore RD, Bone LR, Geller G, Mamon JA, Stokes EJ, Levine DM. Prevalence, detection, and treatment of alcoholism in hospitalised patients. JAMA 1989; 261: 403-7.
(18) Bush B, Shaw S, Cleary P, Delbanco TL, Aronson MD. Screening for alcohol abuse using the CAGE questionnaire. Am J Med 1987; 82:231-5

(19) Cleary PD, Miller M, Bush BT, Warburg MM, Delbanco TL. Aronson MD. Prevalence and recognition of alcohol abuse in a primary care population. Am J Med 1988; 85: 466-71.

(20) D.Liskow B, Campbell J, Nickel EJ, Powell BJ. Validity of the CAGE questionnaire in screening for alcohol dependence in a walk in (triage) clinic. J Stud Alcohol 1995; 56: 277-81.

(21) Isaaeson JH, Butler R, Zacharck M, Tzelepis A. Screening with the Alcohol Use' Disorders Identification Test (AUDIT) in an inner-city population. J Gen Intern Med 1994; 9: 550-3.

(22) Amqdei N, Williams JF, Scale JP, Alvarado ML. Gender differences in medical presentation and detection of patients with a history of alcohol abuse or dependence. J Addict Dis 1996; 15: 19-31.

(23) Gerbert B, Maguire BT, Bleecker T, Coates TJ, McPhee SJ. Primary care physicians and AIDS: attitudinal and structural barriers to care. JAMA 1991; 266: 2837-42.

(24) Lewis DC. The role of internal medicine in addiction medicine. J. Addict Dis 1996; 15:1-17.

(25) Prochaska JO, Di Clemente CC, Norcross JC. In search of how people change: applications to addictive behaviours. Am Psycol 1992; 47: 1102-14.

(26) Samet JH, Rollnick S, Barnes H. Beyond CAGE: a brief clinical approach after detection of substance abuse. Arch Intern Med 1996; 156: 2287-93.

(27) Bien TH, MillerWR, Tonigan JS. Brief interventions for alcohol problems: a review. Addiction 1993; 88: 315-35.

(28) S.Wilk Al, Jensen NM, Havighurst TC. Meta-analysis of randomised control trials addressing brief interventions in heavy alcohol drinkers. J Gen Intern Med 1997; 12: 274-83.

(29) Watson CG, Hancock M, Malovrh P, Gearhart LP, Raden M. A 48 week natural history follow-up of alcoholics who do and do not engage in limited drinking after treatment. J Nerv Ment Dis 1996; 184: 623-7.

(30) Broadening the base of treatment for alcohol problems: report of a study by a committee of Institute of Medicine Washington, D.C.: National Academy Press, 1990.

(31) Hayashida M, Alterman Al, McLellan AT, et al. Comparative effectiveness and costs of inpatient and outpatient detoxification of patients with mild to-moderate alcohol withdrawal syndrome. N Engl J Med 1989; 320: 358-65.

(32) Sullivan JT, Sykora K, Schneiderman J, Naranjo CA, Sellers EM. Assessment of alcohol withdrawal: the revised Clinical Institute Withdrawal Assessment for Alcohol scale (CIWA-Ar). Br J Addict 1989; 84: 1353-7.

(33) Mayo-Smith MF. Pharmacological management of alcohol withdrawal a meta-analysis and evidence-based practice guideline: American Society of Addiction Medicine Working Group on Pharmacological Management of Alcohol Withdrawal. JAMA 1997; 278: 144-51. 
(34) Saitz R, Friedman LS, Mayo-Smith MF. Alcohol withdrawal: a nation-wide survey of inpatient treatment practices. J Gen Intern Med 1995; 10: 479-87.

(35) Litten RZ, Allen J, Fertig J. Pharmacotherapies for alcohol problems: a review of research with focus on developments since 1991. Alcohol Clin Exp Res 1996; 20: 859-76.

(36) Ceccanti M., Attilia M.L., Blum K., Cavaleri G., Franzese A., Sasso G.F., Balducci G.: Gamma-hydroxybutyric acid vs. benzodiazepines: a clinical study in chronic alcoholics. Acta Toxicol., Vol. XVI, n.4: 231-42. 1995.

(37) Project MATCH Research Group. Matching Alcoholism Treatments to client Heterogeneity: Project MATCH post-treatment drinking outcomes. J Stud Alcohol 1997; 58: 7-29.

(38) Volpicelli JR. Naltrexone in alcohol dependence. Lancet 1995; 346: 456.

(39) 0' Malley SS, Croop RS, Wroblewski JM, Labriola DF, Volpicelli JR. Naltrexone in the treatment of alcohol dependence: a combined analysis of two trials. Psychiatr Ann 199'; 25: 681-8.

(40) Whitworth AB, Fischer F, Lesch OM, et al. Comparison of acamprosate and placebo in long-term treatment of alcohol dependence. Lancet 1996 347,1438-47 